\title{
Aprendizaje de la cirugía laparoscópica en Pelvitrainer y en simuladores virtuales
}

\author{
García Galisteo E, Del Rosal Samaniego JM, Baena González V, Santos García Baquero A. \\ Servicio de Urología. Hospital Regional Carlos Haya. Málaga. \\ Fundación IAVANTE. Consejería de Salud. Junta de Andalucía.
}

Actas Urol Esp 2006; 30 (5): 451-456

\section{RESUMEN}

APRENDIZAJE DE LA CIRUGÍA LAPAROSCÓPICA EN PELVITRAINER Y EN SIMULADORES VIRTUALES

En las últimas décadas se han incrementado las técnicas quirúrgicas mínimamente invasivas, como la artroscopia, radiología vascular y en nuestra especialidad la laparoscopia. Con la laparoscopia se ha reducido el daño causado durante la intervención, con la consiguiente disminución del periodo de hospitalización, el dolor postoperatorio y las infecciones. Sin embargo estas técnicas presentan el inconveniente de requerir grandes inversiones en instrumental y un periodo de entrenamiento más largo y costoso. En el siguiente capítulo repasamos el proceso de aprendizaje laparoscópico realizado en el pelvitrainer y en el simulador virtual por parte de los residentes de nuestro Servicio de Urología.

Palabras clave: Laparoscopia. Aprendizaje. Simuladores virtuales.

\section{ABSTRACT}

LAPAROSCOPIC SURGERY TRAINING IN PELVITRAINER AND VIRTUAL SIMULATORS

Over the last few decades, there has been a rise in the number of minimally invasive techniques, such as arthroscopy, vascular radiology and our speciality, laparoscopy. Laparoscopy has resulted in a reduction in the damage caused during intervention, with the subsequent reduction in hospital stay, postoperative pain and infections. However, one disadvantage of these techniques is that they require a large investment in instruments and a long and costly training period. In the following chapter, we describe the laparoscopic training process of the medical residents from our Urology Service in the pelvitrainer and virtual simulator.

Keywords: Laparoscopy. Training. Virtual simulators.

$\mathrm{H}$ istóricamente el urólogo ha aprendido a realizar las diferentes técnicas quirúrgicas durante intervenciones en pacientes reales con la supervisión de un urólogo experto. Esto sin embargo no es admisible en el campo de la laparoscopia dado que los procedimientos quirúrgicos requieren en general mayor tiempo operatorio y mayor coste de material, por tanto el urólogo debe entrenar en otro espacio que no sea el paciente para acortar en lo posible el tiempo operatorio y la morbilidad sobre el paciente.

Aunque no existen unas pautas únicas de aprendizaje laparoscópico, creemos que se deben de adquirir los conocimientos necesarios de forma secuencial de la siguiente manera:

1. Estudio para la adquisición de conocimientos teóricos a través de libros, bibliografía, etc.

2. Ejercicios en simuladores bien en pelvitrainer o simuladores virtuales, donde se debe dominar el instrumental y ejercitar ciertos gestos quirúrgicos (disección, corte, sutura, anudado, etc).

3. Prácticas en animales de experimentación donde se deben realizar intervenciones complejas siguiendo los mismos pasos que se harían en el paciente real. 
4. Demostraciones prácticas donde se vería intervenciones realizadas por urólogos expertos en cirugía laparoscópica, en directo o videoconferencia lo que nos permite ver detalles técnicos, ver como solucionan situaciones imprevistas y complicaciones surgidas durante la cirugia.

5. Enseñanza en medios audiovisuales con el visionado de diferentes intervenciones filmadas, videotecas, etc.

6. Y por último la supervisión durante las primeras intervenciones en pacientes reales por un urólogo laparoscopista experto ${ }^{1}$.

En este capítulo nos vamos a centrar en los ejercicios básicos que podemos realizar en los simuladores (pelvitrainer) y simuladores virtuales. Para llegar a dominar la cirugía laparoscópica el urólogo debe de desarrollar una serie de habilidades como la visión bidimensional, habituarse al material laparoscópico, etc; para ello debe desarrollar un plan de entrenamiento donde se comenzará realizando ejercicios en un simulador o pelvitrainer y una vez habituado a estos ejercicios se puede pasar a realizar determinados procedimientos laparoscópicos en el animal de experimentación. A continuación vamos a ir desarrollando una serie de ejercicios para lograr un correcto aprendizaje en la cirugia laparoscópica.

En el simulador podemos adquirir el manejo del instrumental y equipos quirúrgicos, adaptación a la imagen bidimensional y aprendizaje de suturas laparoscópicas ${ }^{2}$. Estos ejercicios se deben hacer inicialmente con visión directa y posteriormente a través de un monitor.

Ejercicio 1: Manejo del instrumental: Deberemos conocer las características y modo de empleo de los trócares y de las agujas de veress, introducirlos por la cubierta superior del simulador comprendiendo su mecanismo de acción y sistema de seguridad. Introducción de las pinzas y tijeras a su través y habituarse a su manejo. Se debe colocar el trocar de la óptica entre ambas manos para que los movimientos y la coordinación vista-mano sea la mejor posible (Fig. 1).

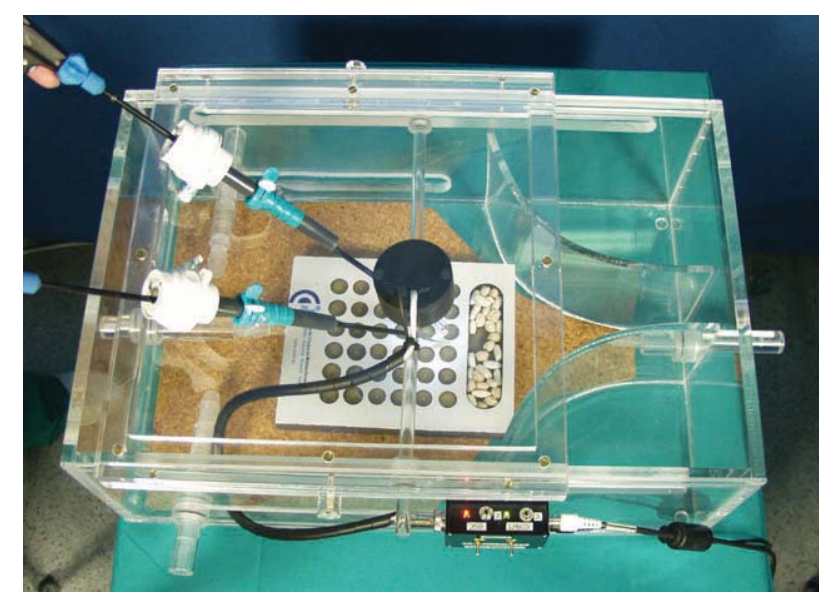

FIGURA 1. Pelvitrainer. Introducción de los trócares e instrumental por la cubierta superior.

Ejercicio 2: Manejo de pequeños objetos (garbanzos, habichelas, etc) haciendo prensa con diferentes tipos de pinzas e intercambiándoselos de pinza para así habituarse al manejo de ambas manos, y tener una coordinación entre ambas manos. Se debe ejercitar la rotación de las pinzas y tijeras para aprovechar las posibilidades del instrumental (Fig. 2).

Ejercicio 3: Disección con pinza y tijera de diferentes estructuras: látex o tejidos orgánicos ex vivo (piel de pollo, estómago, riñón con pedículo vascular, vejiga urinaria, etc). Este ejercicio nos permite perfeccionar la coordinación entre ambas manos e incluso lo que es más importante, la coordinación con el ayudante haciendole participe en los ejercicios lo cual nos ayudará cuando estemos realizando las intervenciones en el animal de experimentación o en el paciente real (Figs. 3 y 4).

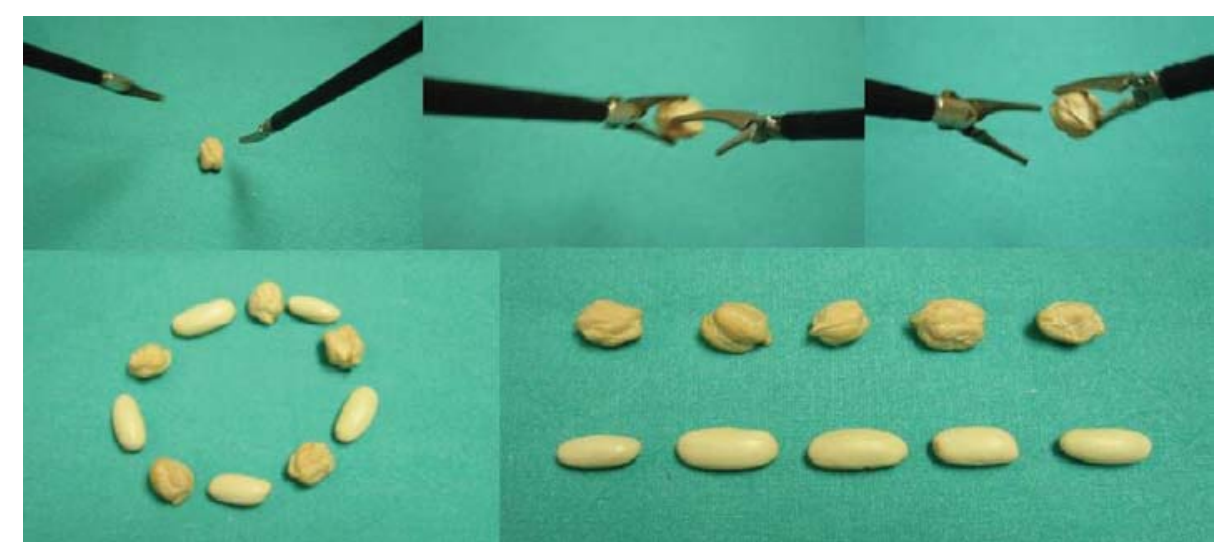

FIGURA 2. Manejo de pequeños objetos. 


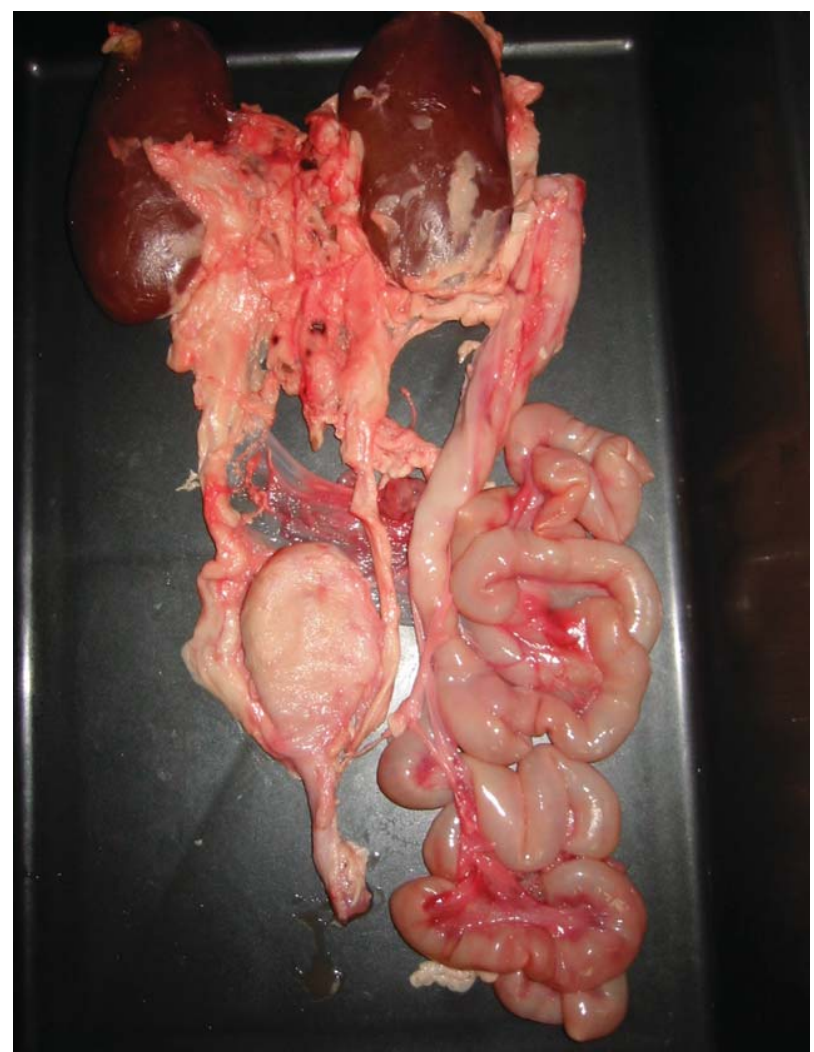

FIGURA 3. Bloque de visceras donde se pueden realizar multitud de ejercicios.

Ejercicio 4: Realización de suturas laparoscópicas. Se iniciará el ejercicio introduciendo las suturas a través de los trócares, se colocarán las agujas correctamente en el porta para posteriormente realizar el paso de la aguja y el anudado laparoscópico, se debe realizar un primer nudo doble y posteriormente nudos simples (Fig. 5). Nosotros recomendamos la realización de la sutura laparoscópica manual al igual que la realizamos en la cirugía convencional y que posteriormente, cuando se domine correctamente la técnica de anudado el cirujano emplee si lo desea otro tipo de métodos de sutura: endostich, grapas reabsorvibles (Fig. 6), puntos montados, etc; que disminuya el tiempo operatorio. La realización de este tipo de suturas tiene el inconveniente de aumentar la curva de aprendizaje y el tiempo quirúrgico aunque va a proporcionar al cirujano suficiente habilidad para solucionar problemas que le pudieran ocurrir en la práctica clínica. Se deben realizar suturas con puntos sueltos y suturas continuas. Hay que hacer hincapié en la realización de puntos con ambas manos para intentar ser lo más ambidiestros posible.

Ejercicio 5: Anastomosis uretero-vesical: Este ejercicio se puede realizar con piezas de animales que incluyan los uréteres y la vejiga (Fig. 7). Este ejercicio nos ayudará a la realización de futuras pieloplastias. Es más fácil ejercitarse en la realización de una anastomosis uretero-vesical ya que es muy difícil encontrar una pelvis renal dilatada en el cerdo. Debemos introducir un catéter en el uréter, al igual que lo haríamos en la cirugía abierta, que nos ayude a la realización de la sutura que debemos practicar con puntos sueltos y sutura continua.

Ejercicio 6: Anastomosis uretrovesical. El cerdo castrado prácticamente no tiene próstata pero nosotros podemos realizar la sección del 1-2 cm de uretra cercana al cuello vesical para posteriormente practicar la sutura uretro-vesical, con puntos sueltos y con suturas continuas. Este ejercicio debe reservarse para cuando el equipo quirúrgico tenga bastante experiencia y coordinación (Fig. 8).

Los ejercicios previos son algunos de los muchos que se pueden realizar y que dependen del procedimiento que se quiera entrenar (nefrectomía, prostatectomía, linfadenectomía, cistectomías con neovejigas, etc) y de la imaginación del cirujano. Lo que es incuestionable es que cuanto más se practiquen estos ejercicios y se coordine al equipo quirúrgico, menor será el tiempo operatorio y mejores serán los resultados en el paciente real.

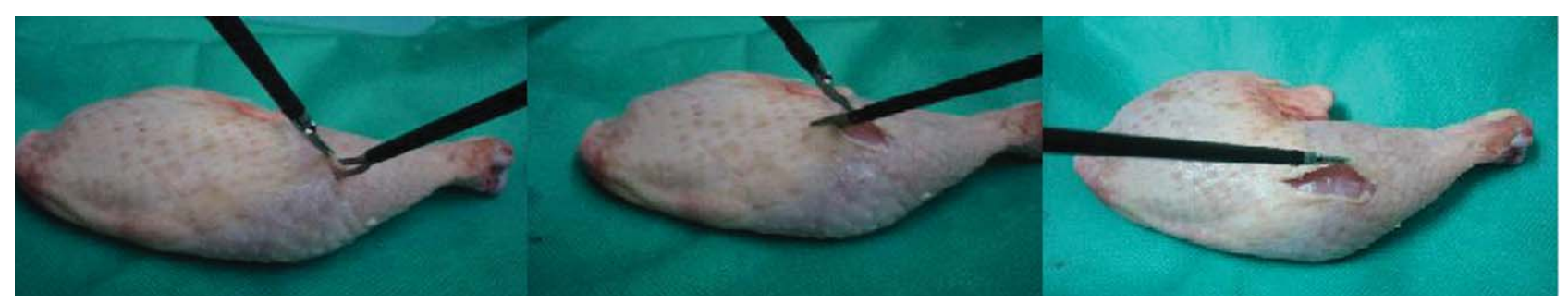

FIGURA 4. Disección de piel de pollo. 


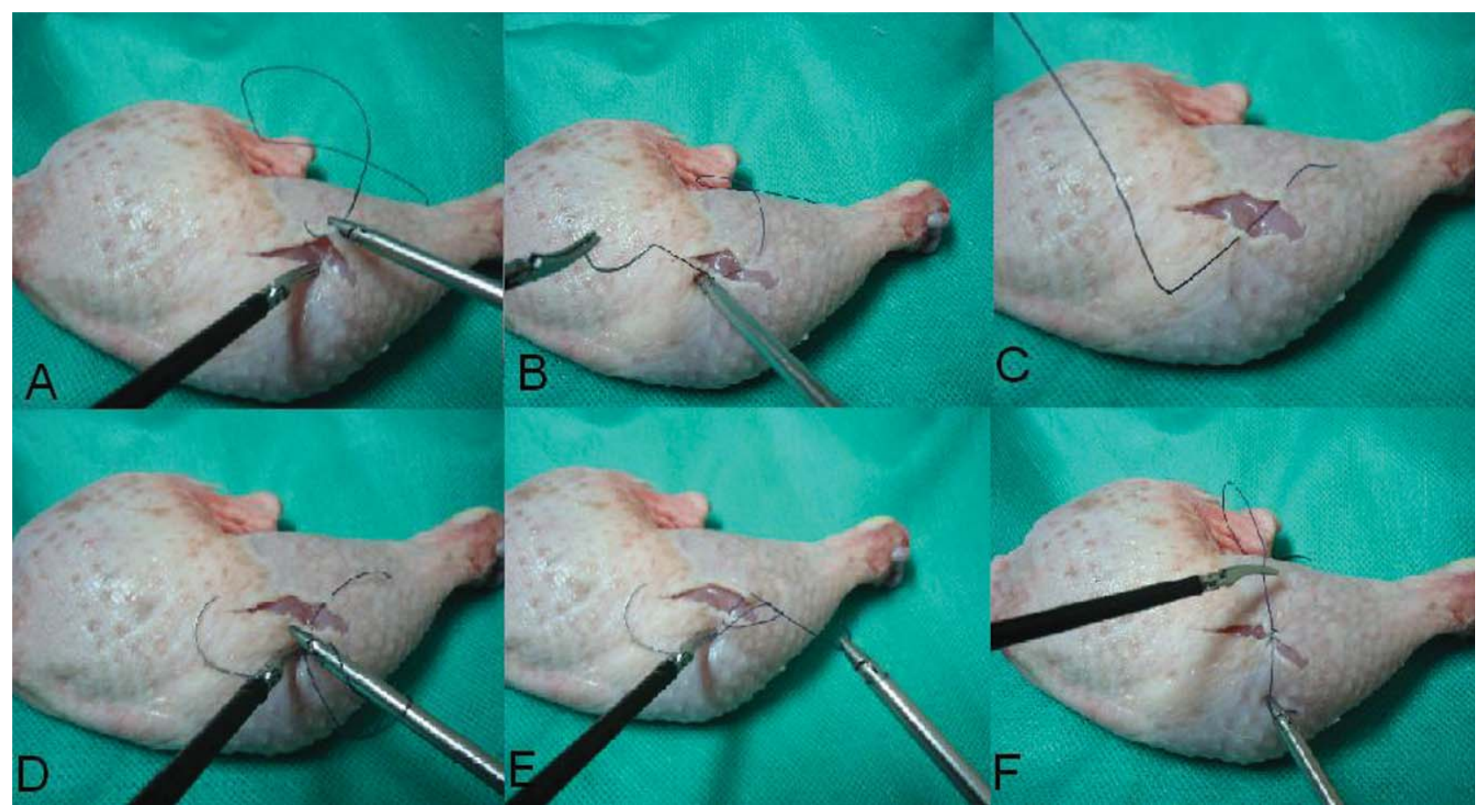

FIGURA 5. Anudado laparoscópico: A y B) Paso de aguja por ambos bordes. C) Paso del hilo hasta dejar un cabo corto de unos $2 \mathrm{~cm}$. D) Realización de un nudo doble. E) Se baja el nudo. F) Se tracciona equidistantemente hasta apretar el nudo.

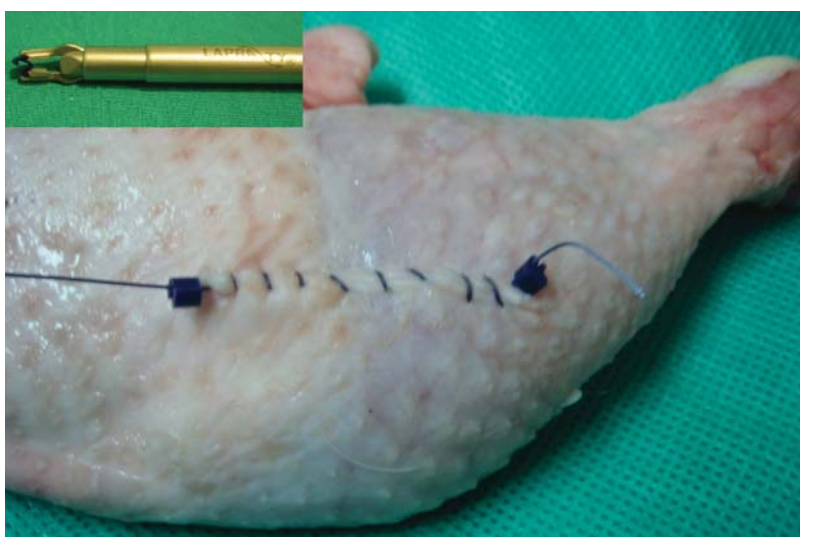

FIGURA 6. Sutura continua anudada con grapas de vicryl.

\section{SIMULADORES QUIRÚRGICOS VIRTUALES}

En los últimos años el desarrollo de la informática gráfica ha permitido desarrollar entornos visuales que van a ir permitiendo el entrenamiento de determinadas técnicas quirúrgicas en un ambiente virtual.

Un buen simulador quirúrgico debe de cumplir una serie de características: debe mostrar los órganos internos de manera realista, que éstos órganos respondan a las interacciones con el cirujano, por ejemplo deformándose y que respondan mediante modificaciones estructurales a acciones quirúrgicas habituales como cortes, cauterización o sutura (Figs. 9, 10 y 11).

En función de que consigan estos objetivos los simuladores quirúrgicos virtuales se agrupan en tres generaciones:

Primera generación: Únicamente consideran la naturaleza geométrica de las estructuras anatómicas, pero no nos permiten interaccionar con ellos.

Segunda generación: Permiten una cierta interacción con las estructuras anatómicas.

Tercera generación: Permiten una interacción con las estructuras y además tienen en cuenta la naturaleza funcional de los órganos.

Actualmente la mayoría de los simuladores virtuales están incluidos en los de segunda generación y dentro de ellos existen cuatro niveles de complejidad:

- Simuladores de aguja que disponen de objetos visuales simples con un mínimo grado de libertad.

- Simuladores que permiten una exploración endoscópica o instalación de catéteres.

- Simuladores para el entrenamiento en una tarea, que disponen de uno o dos instrumentos que interaccionen con el entorno virtual. 


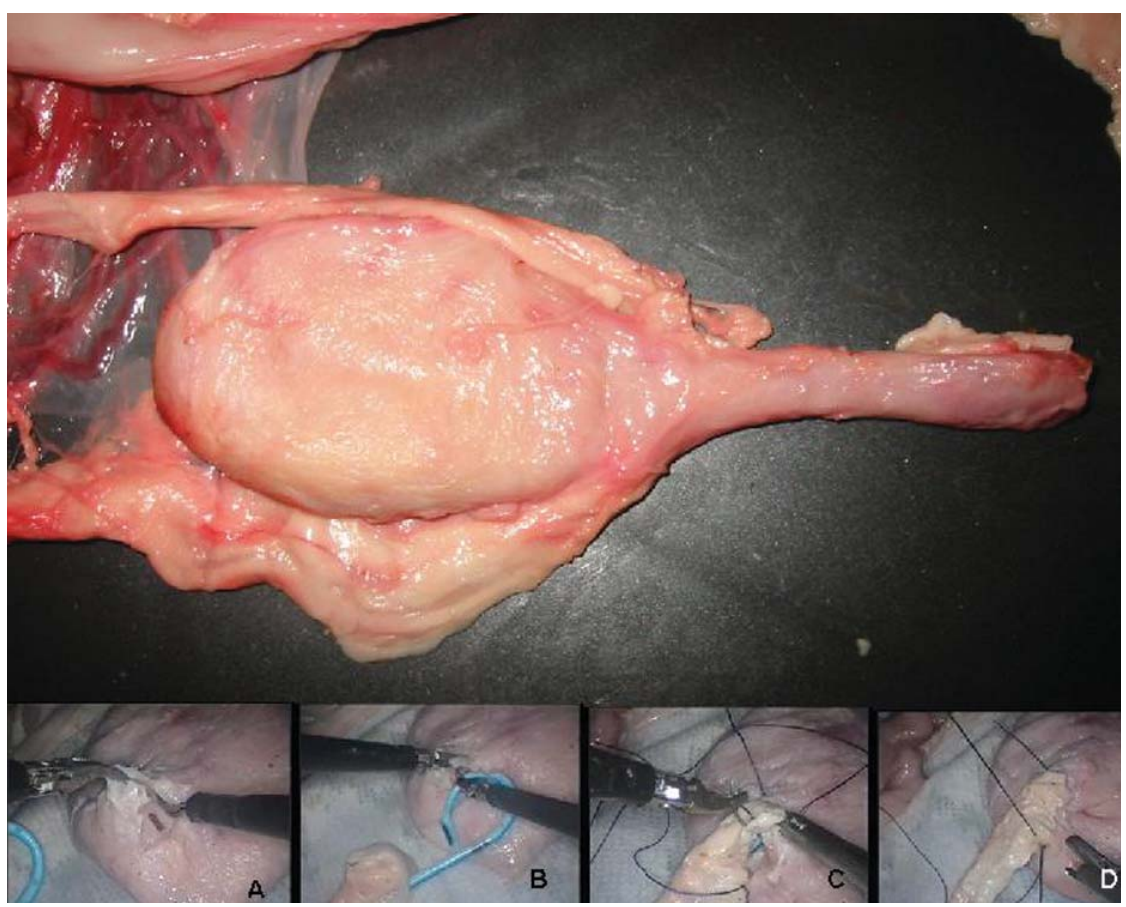

FIGURA 7. Vejiga urinaria con uréter. A) Apertura vesical por capas. B) Introdución de catéter doble J. C y D) Sutura continua urétero-vesical.
- Simuladores completos: permiten el entrenamiento en determinados procedimientos quirúrgicos laparoscópicos, artroscopia, ginecología o cirugía intraocular $^{3}$ (Figs. 12 y 13).

El problema actual es que conforme los simuladores son más complejos la imagen y la interrelación con el usuario es menos realista y no traducen el comportamiento real de los tejidos a nuestra actuación ya que estos simuladores requieren ordenadores y programas informáticos más grandes y complejos. Actualmente con los simuladores virtuales se pueden realizar prácticamente la mayoría de los ejercicios básicos (coordinación mano-

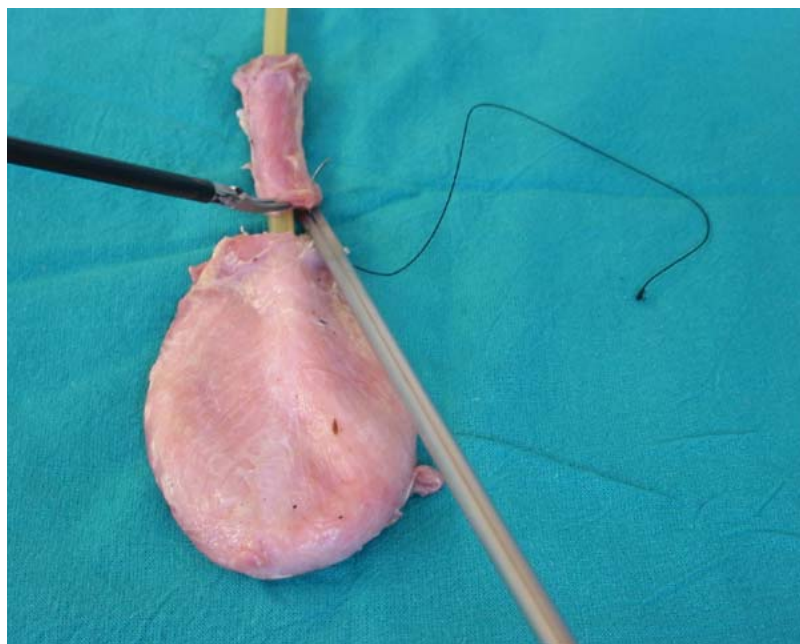

FIGURA 8. Sutura uretro-vesical.

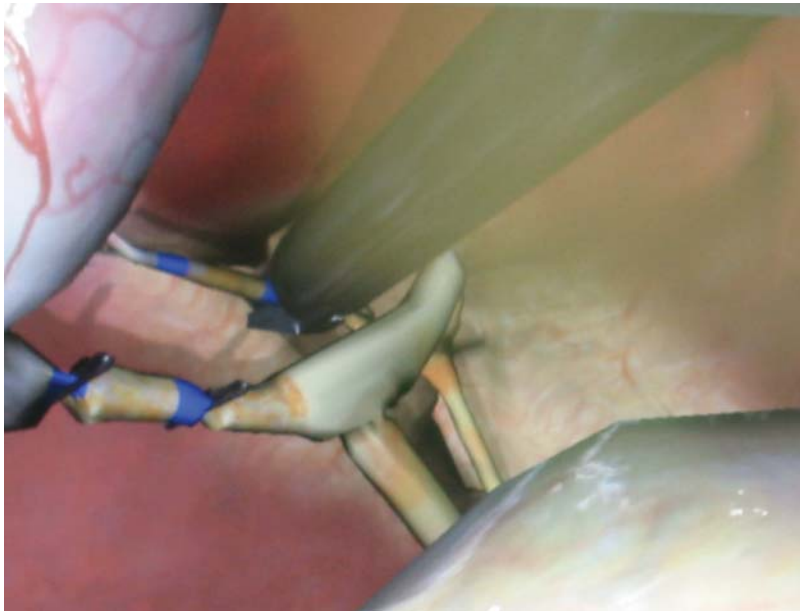

FIGURA 10. Ejercicios de corte y disección con simulador virtual.

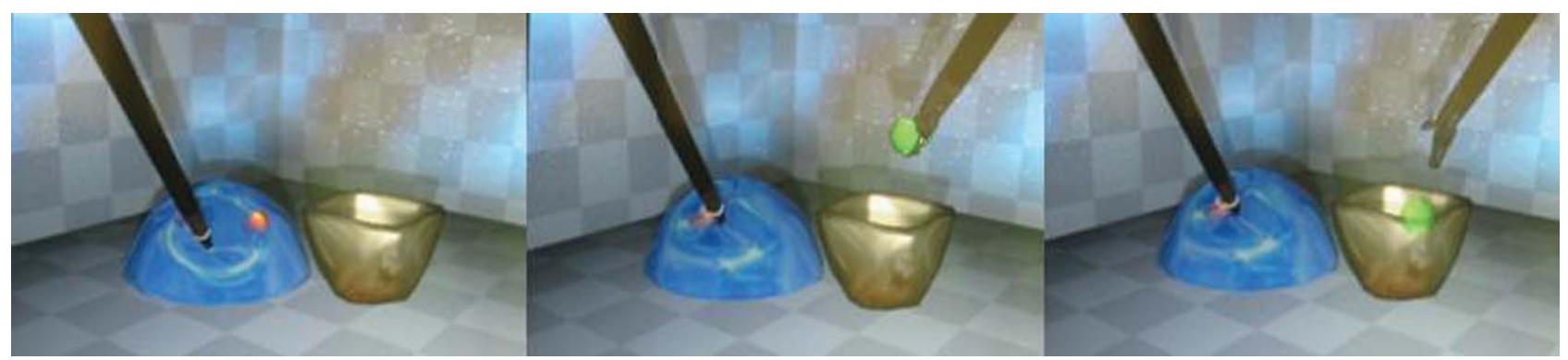

FIGURA 9. Ejercicios básicos de coordinación con simulador virtual. 


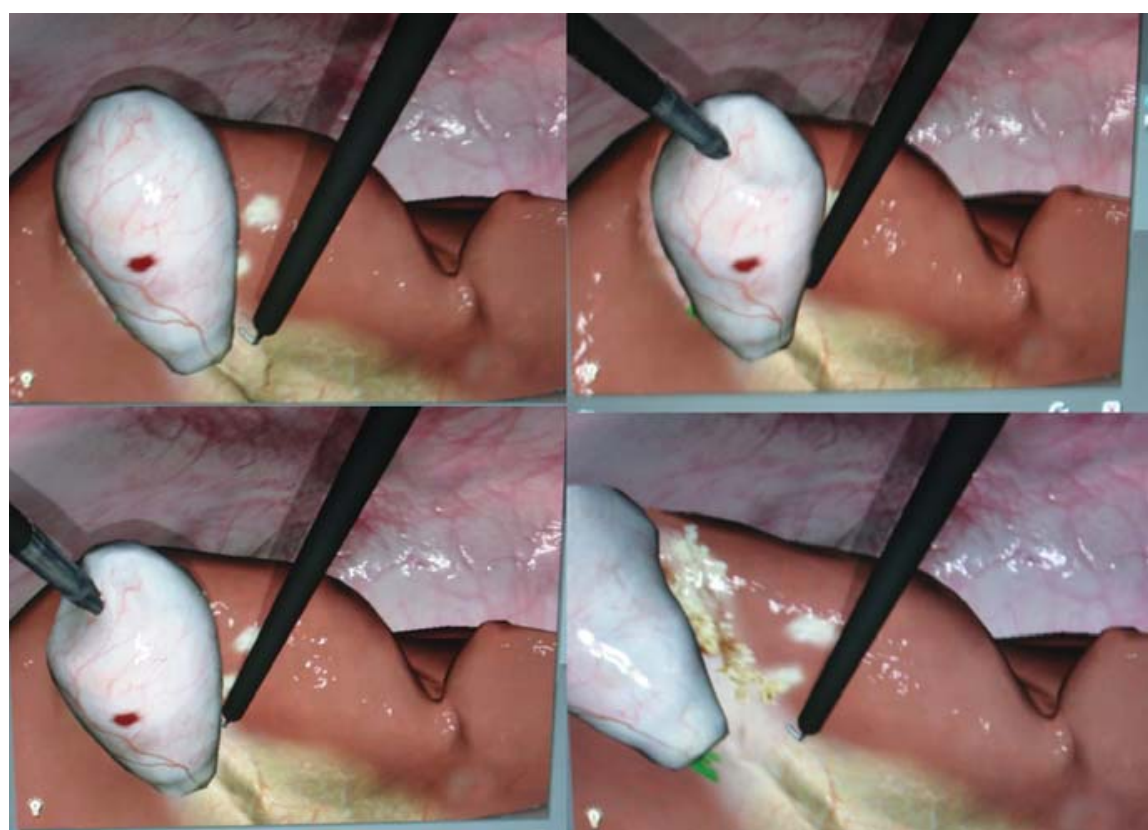

FIGURA 11. Colecistectomía laparoscópica virtual.

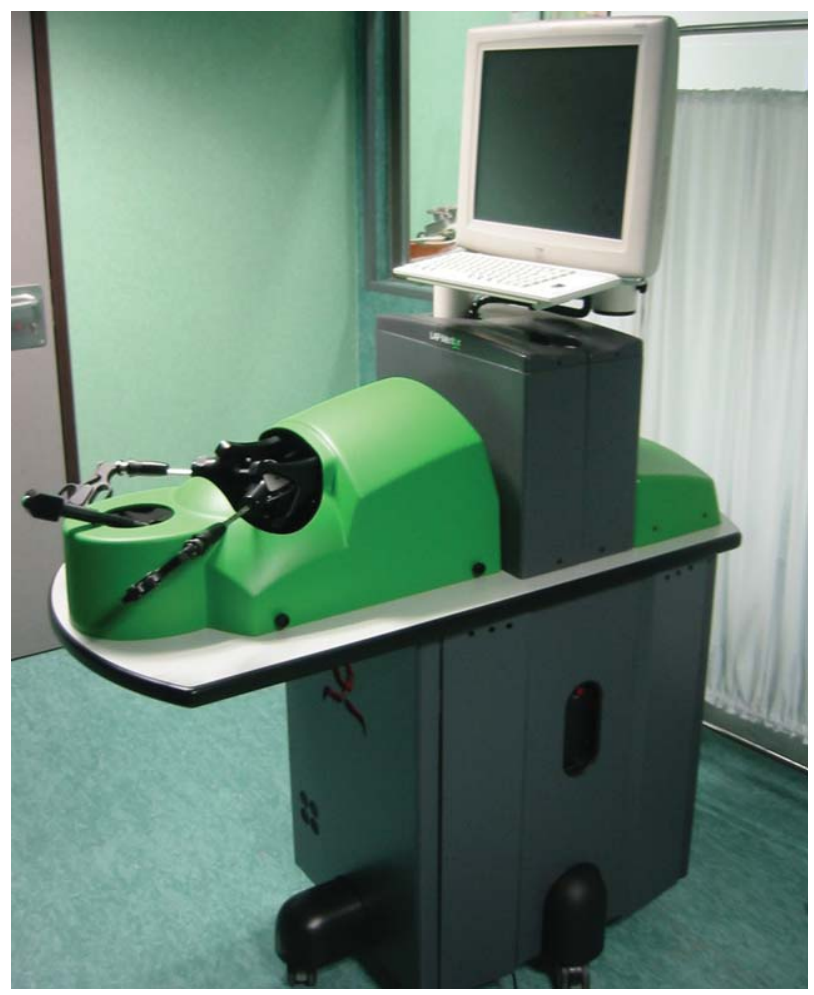

FIGURA 12. Simulador virtual LAP Mentor.

vista; corte, cauterización, anudado, etc.) que se pueden realizar en un pelvitrainer común sin embargo quedan por desarrollar ejercicios complejos como la realización de nefrectomías, adre- nalectomías, suturas uretrovesicales que requieren ordenadores de mayor potencia, no obstante se están dando los pasos necesarios para que en los próximos años podamos disponer en nuestra especialidad de simuladores que nos permitan reproducir alguno de los procedimientos quirúrgicos que ahora requieren el empleo de animales de experimentación.

Agradecimiento a IAVANTE (Fundación para el Avance Tecnológico y Entrenamiento Profesional) a CCMI (Centro de Cirugía Mínimamente Invasiva. Cáceres) y a Medical Simulation por su ayuda en la realización de este capítulo.

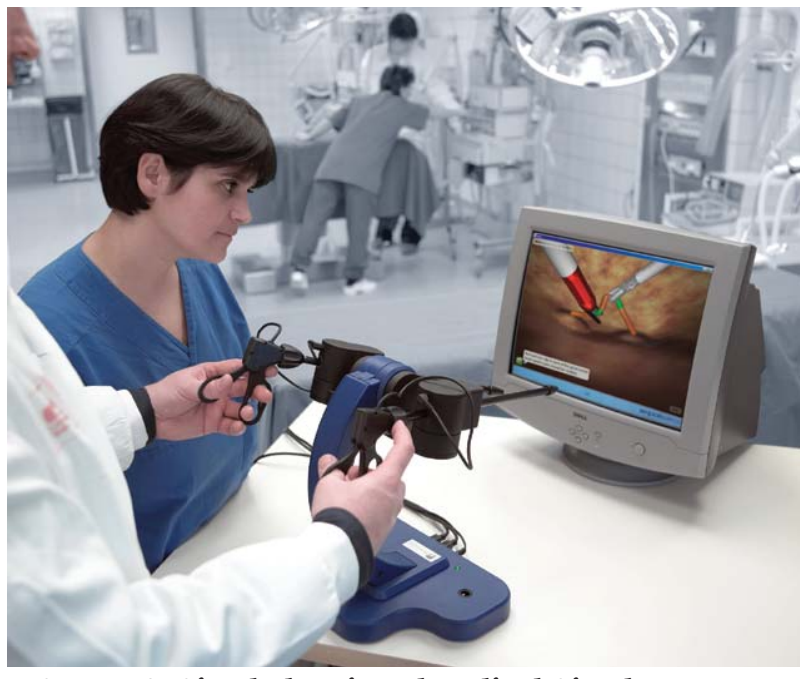

FIGURA 13. Simulador virtual Medical Simulator.

\section{REFERENCIAS}

1. Usón J, Sánchez FM, Pascual S, Climent S. Formación en cirugía laparoscópica paso a paso. Cáceres: Centro de Cirugia Minimamente Invasiva. 2005.

2. Sánchez FM, Gómez EJ, Pagador JB, Monserrat C, Pascual S, Alcañiz M, et al. Integración de la Tecnología de Simulación Quirúrgica en el Programa de Aprendizaje de Cirugía de Mínima Invasión. Informática y Salud. 2004;47:9-14.

3. Monserrat C, López O, Alcañiz M. Estado del Arte en simulación quirúrgica. Informática y Salud. 2004;47:15-22.

Dr. E. García Galisteo

E-mail: eggalisteo@yahoo.es 